\title{
Temperatura do ar e emissões urbanas em região industrial de São Paulo, Brasil
}

\author{
María Cleofé Valverdea*, Humberto de Paiva Junior \\ ${ }^{a}$ Programa de Pós-graduação em Ciência e Tecnologia Ambiental, Universidade Federal do ABC, São Paulo, Santo André, 09210-580, \\ Brasil. *maria.brambila@ufabc.edu.br \\ ${ }^{b}$ Laboratório de Modelagem Ambiental e Urbana, Universidade Federal do ABC, São Paulo, Santo André, 09210-580, Brasil.
}

Recebido: 19 março 2018 / Aceito: 20 maio 2018 / Publicado online: 28 maio 2018

\begin{abstract}
Resumo
$\mathrm{O}$ objeto dessa pesquisa é analisar a relação entre a temperatura do ar e as emissões veiculares de $\mathrm{CO}_{2}$ na área urbana do $\mathrm{ABC}$ Paulista, localizada no sudeste da Região Metropolitana de São Paulo. Conjuntamente são analisadas as projeções de temperatura de dois modelos climáticos utilizados nos relatórios do Intergovernmental Panel on Climate Change (IPCC). Foram utilizados dados de temperatura do ar na escala temporal horária e mensal, e a estimativa de emissões veiculares foi realizada através do modelo de simulação de tráfego Equilibre Multimodal/Multimodal Equilibrium (EMME). Os resultados mostram que as áreas com as temperaturas mais altas se localizam no setor norte do ABC Paulista, indicando uma ilha de calor de média intensidade $\left(3,6{ }^{\circ} \mathrm{C}\right)$. Este setor também se destacou como o de maior emissão veicular de $\mathrm{CO}_{2} \mathrm{em}$ ton $/ \mathrm{km}$, por apresentar extensas e movimentadas vias de tráfego. As projeções dos modelos climáticos, apesar das incertezas, indicaram um cenário de dias e noites mais quentes, com um aumento de $6,3^{\circ} \mathrm{C}$ na temperatura máxima anual para o período de 2071-2100. A distribuição geográfica das áreas de altas temperaturas e emissões de $\mathrm{CO}_{2}$ sugerem a contribuição do tráfego urbano para o aumento da temperatura no ABC Paulista, a qual tende a crescer conforme projeção do modelo do IPCC.
\end{abstract}

Palavras-chave: Região do ABC Paulista, simulação de tráfego, emissões de fontes móveis, ilha de calor.

\section{Air temperature and urban emissions in an industrial region of São Paulo, Brazil}

\begin{abstract}
The objective of this research is the analysis of the relationship between air temperature and vehicles $\mathrm{CO}_{2}$ emissions in $\mathrm{ABC}$ Paulista urban area, located in the southeast of São Paulo Metropolitan Region. Beside, the temperature projections from two IPCC climatic models were analyzed. The air temperature data in the hourly and monthly time scale were used to identify the intensity of the heat islands. Estimation of vehicular emissions was performed using the EMME traffic simulation model. The results showed that the areas with the highest temperatures are in the northern sector of $\mathrm{ABC}$ Paulista, indicating a heat island of medium intensity $\left(3.6{ }^{\circ} \mathrm{C}\right.$ ). This sector also stood out as the one with the highest $\mathrm{CO}_{2}$ road emission (ton/km) because it presents the most extensive and busiest traffic routes. The climate model projections, despite the uncertainties, indicated a scenario of warmer days and nights, with a rise of $6.3{ }^{\circ} \mathrm{C}$ in the maximum annual temperature for the period 2071-2100. The analysis of the geographic distribution of areas with high temperature and high $\mathrm{CO}_{2}$ emissions indicate a contribution of urban traffic emissions to the elevation in air temperature in ABC Paulista, which tends to increase based on IPCC model projection.
\end{abstract}

Keywords: ABC Paulista Region, transport simulation, mobile emissions, air temperature.

\section{Introdução}

Uma das principais alterações climáticas em áreas urbanas está relacionada com o aumento da temperatura do ar em locais próximos à avenidas de grande circulação de tráfego e complexos industriais. O resultado é a formação de ilhas de calor (IC).

Segundo Oke (1987) o principal fator da formação de uma IC é a concentração relativamente alta de fontes de calor nas cidades. O processo de urbanização leva a construção de prédios e habitações, diminuição das áreas verdes, maior estabelecimento de indústrias, crescimento da frota veicular para transporte da população, junto com as emissões de poluentes. Estes fatores têm gerado alterações consideráveis na absorção da radiação que chega a superfície e na circulação local.

Em centros urbanos a temperatura média anual é tipicamente mais alta que a de suas redondezas, e em alguns dias esse contraste pode atingir cerca de $10^{\circ} \mathrm{C}$ ou mais (Stull, 1988). O contraste de temperatura forma uma circulação convectiva que contribui para a concentração de poluentes sobre as grandes cidades (Oke, 1987).

Estudos recentes sobre ilhas de calor em cidade de mediano 
e pequeno porte no estado de São Paulo têm encontrado intensidades de ilhas de calor de até $4,5{ }^{\circ} \mathrm{C}$ entre pontos intraurbanos, para dias específicos, na cidade de Rancharia, São Paulo (Porangaba et al., 2017) e de $2,5^{\circ} \mathrm{C}$ para a região central da cidade de São Carlos - SP quando comparada com a área rural (Vasconcelos et al., 2013).

Diferenças nas intensidades da amplitude térmica que pode caracterizar uma ilha de calor varia de local para local e depende preferencialmente do tipo de material construtivo e da porcentagem de área vegetal. No entanto, em áreas urbanas também tem outro fator a ser considerado que é a concentração de poluentes vindas de fontes emissoras (móveis e fixas) que também influencia no balanço de radiação e consequentemente na temperatura do ar.

De acordo com Martins e Andrade (2008), os poluentes ou aerossóis também afetam o balanço da irradiação, pois, poluentes ou aerossóis tem a capacidade de absorver e emitir maior radiação ultravioleta o que reflete numa elevação da temperatura, em especial, pela presença significativa de ozônio $\left(\mathrm{O}_{3}\right)$ e $\mathrm{CO}_{2}$.

Segundo a Companhia Ambiental do Estado de São Paulo - CETESB (2016) em 2015, na RMSP, as fontes móveis e fixas foram responsáveis pela emissão para a atmosfera de aproximadamente 167 mil t/ano de monóxido de carbono, 44 mil t/ano de hidrocarbonetos, $80 \mathrm{mil} \mathrm{t/ano} \mathrm{de} \mathrm{óxidos} \mathrm{de}$ nitrogênio, 5 mil t/ano de material particulado e 7 mil t/ano de óxidos de enxofre. Desses totais, os veículos são responsáveis por $97 \%$ das emissões de $\mathrm{CO}, 79 \%$ de $\mathrm{HC}, 68 \%$ de NOx, $40 \%$ de Material Particulado (MP) e $22 \%$ de SOx (CETESB, 2016).

No estado de São Paulo, a qualidade do ar é diretamente influenciada pela distribuição e intensidade das emissões de poluentes atmosféricos de origem veicular e industrial. Exercem papel fundamental a topografia e as condições meteorológicas, que se alteram de modo significativo nas várias regiões do Estado (CETESB, 2016).

A região do $\mathrm{ABC}$ tem uma grande contribuição nessas porcentagens, com suas avenidas e rodovias. Além disso, há a contribuição das indústrias, como o polo petroquímico de Capuava, o setor do plástico, e a indústria automotiva, com os setores: metalúrgico, mecânico e eletrônico.

Apesar de diversos estudos para entender a circulação e poluição atmosférica na cidade de São Paulo (Ccoyllo e Andrade, 2002; Freitas et al., 2007; Boian e Andrade, 2012) poucos tratam das singularidades da Região do Grande ABC e da sua relevância como fonte de poluentes.

No contexto de mudanças climáticas, as temperaturas em áreas urbanas podem se elevar ainda mais considerando a alteração do padrão de circulação e da falta de chuvas. No entanto, não é fácil distinguir o que é a contribuição da ação antrópica em uma área urbana e a mudança do padrão global da atmosfera que produz o aumento da temperatura.

Em relação a cenários futuros de mudanças climáticas, os modelos climáticos servem como uma ferramenta primordial para inferir diferentes cenários de aumento da temperatura considerando os cenários de emissões utilizados nos relatórios do IPCC (IPCC, 2013). Modelos Climáticos são uma representação matemática que utiliza como base as equações físicas da primeira e segunda Lei de Newton para simular os processos naturais que possam afetar o tempo e clima em várias escalas de tempo. Os modelos sempre estão em aprimoramento e têm mostrado grandes avanços nos últimos anos em termos da representação de processos e fenômenos críticos para estudo das mudanças climáticas globais, seus impactos e mitigação (Randal et al., 2007).

Esta pesquisa tem como objetivo principal analisar a variabilidade espacial da temperatura na área urbana do $\mathrm{ABC}$ Paulista e sua relação com as emissões veiculares de $\mathrm{CO}_{2}$, além de utilizar dois modelos climáticos para analisar a temperatura do ar para dois cenários de emissões: RCP2.6 e RCP8.5.

\section{Material e Métodos}

A região do Grande $\mathrm{ABC}$ Paulista está localizada no sudeste da Região Metropolitana de São Paulo (RMSP) e é composta pelos municípios de Santo André, São Bernardo do Campo, São Caetano do Sul, Diadema, Mauá, Ribeirão Pires e Rio Grande da Serra (Fig. 1A). Possui uma população de 2.549.135 habitantes, um território de $841 \mathrm{~km}^{2}$ (IBGE, 2010) e é considerado o maior polo industrial da RMSP, apresentando caraterísticas próprias, pois parte de seu território apresenta uma massa urbana densamente construída com grande densidade populacional e outra parte constituída por áreas de proteção ambiental. Este contraste do espaço já possibilita inferir caraterísticas diferenciadas do clima da região. Além disso, a parte urbana do ABC Paulista apresenta uma frota significativa de veículos automotores e possui um forte caráter industrial. Temos como exemplo, na divisa de Santo André com Mauá, o Polo Petroquímico de Capuava, constituído pela Refinaria de Capuava.

Para a identificação das caraterísticas térmicas e ilhas de calor atmosféricas na região de estudo foram utilizadas dados de temperatura do ar de nove estações meteorológicas automáticas distribuídas na região do ABC Paulista (Fig. 1B), as quais são administradas pela CETESB, pelo Serviço Municipal de Saneamento Ambiental de Santo André (SEMASA), e pelo Sistema de Alerta a Inundações de São Paulo (SAISP). No município de Santo André se localizam cinco (05) estações, uma (01) estação em São Caetano do Sul e em Mauá, e duas (02) em São Bernardo do Campo.

Inicialmente se realizou uma análise espacial da temperatura média mensal, tendo como base os dados obtidos das estações meteorológicas. Posteriormente, para a identificação de possíveis núcleos de ilhas de calor utilizou-se o cálculo das diferenças de temperatura $(\Delta \mathrm{T})$ entre os diferentes locais onde se encontram as estações meteorológicas e foram realizadas diferentes combinações de $\Delta \mathrm{T}$ a fim de identificar as áreas de maior variação térmica. $\mathrm{O} \Delta \mathrm{T}$ mede a intensidade da variação térmica entre dois pontos e foi determinado pela seguinte expressão (Oke, 1987):

$$
\Delta \mathrm{T}_{\mathrm{h}}=\mathrm{TU}_{(\mathrm{h})}-\mathrm{TR}_{(\mathrm{h})}
$$

Onde: $\mathrm{TU}_{(\mathrm{h})}$ : Temperatura do ar horária na área urbana1; $\mathrm{TR}_{(\mathrm{h})}$ : Temperatura do ar horária na área urbana 2.

Com base no cálculo do $\Delta \mathrm{T}$ em escala horária foi construído o ciclo diurno da temperatura do ar com o intuito de verificar se a ilha de calor é um fenômeno noturno, diurno, ou vespertino, assim como identificar a sua intensidade média, segundo a classificação de Garcia (1996): Fraca magnitude $\left(0,1{ }^{\circ} \mathrm{C}\right.$ até $\left.2{ }^{\circ} \mathrm{C}\right)$; Média Magnitude $\left(2{ }^{\circ} \mathrm{C}\right.$ até $\left.4{ }^{\circ} \mathrm{C}\right)$; Forte 
Magnitude $\left(4{ }^{\circ} \mathrm{C}\right.$ até $\left.6{ }^{\circ} \mathrm{C}\right)$; Muito Forte $\left(>6{ }^{\circ} \mathrm{C}\right)$ e Ilha de Frescor $\left(<0^{\circ} \mathrm{C}\right)$.

Para o cálculo das emissões das fontes móveis, no caso do ABC Paulista, considerou-se um sistema aberto porque ele está inserido na RMSP onde uma parcela significativa das emissões que ocorrem em seu território é gerada por veículos de passagem, e foi dada prioridade ao problema de identificação das vias de maior potencial de emissão de gases de efeito estufa (GEE). Quanto à ferramenta, o método de modelagem escolhido foi a macro simulação de tráfego empregando um modelo de cálculo de emissões sensível à velocidade de tráfego. Essa escolha foi realizada com base na disponibilidade de um modelo de alocação de tráfego consolidado e validado para a região desenvolvido pela Companhia Paulista de Trens Metropolitanos (CPTM) em 2010 usando o software Equilibre Multimodal/Multimodal Equilibrium (EMME).

O modelo EMME gera resultados de fluxo e velocidade de tráfego para diversos cenários e horizontes de planejamento. Esse trabalho empregou os dados do cenário de 2014 que representa o sistema atual de transportes públicos e individuais da região, bem como a demanda do sistema.
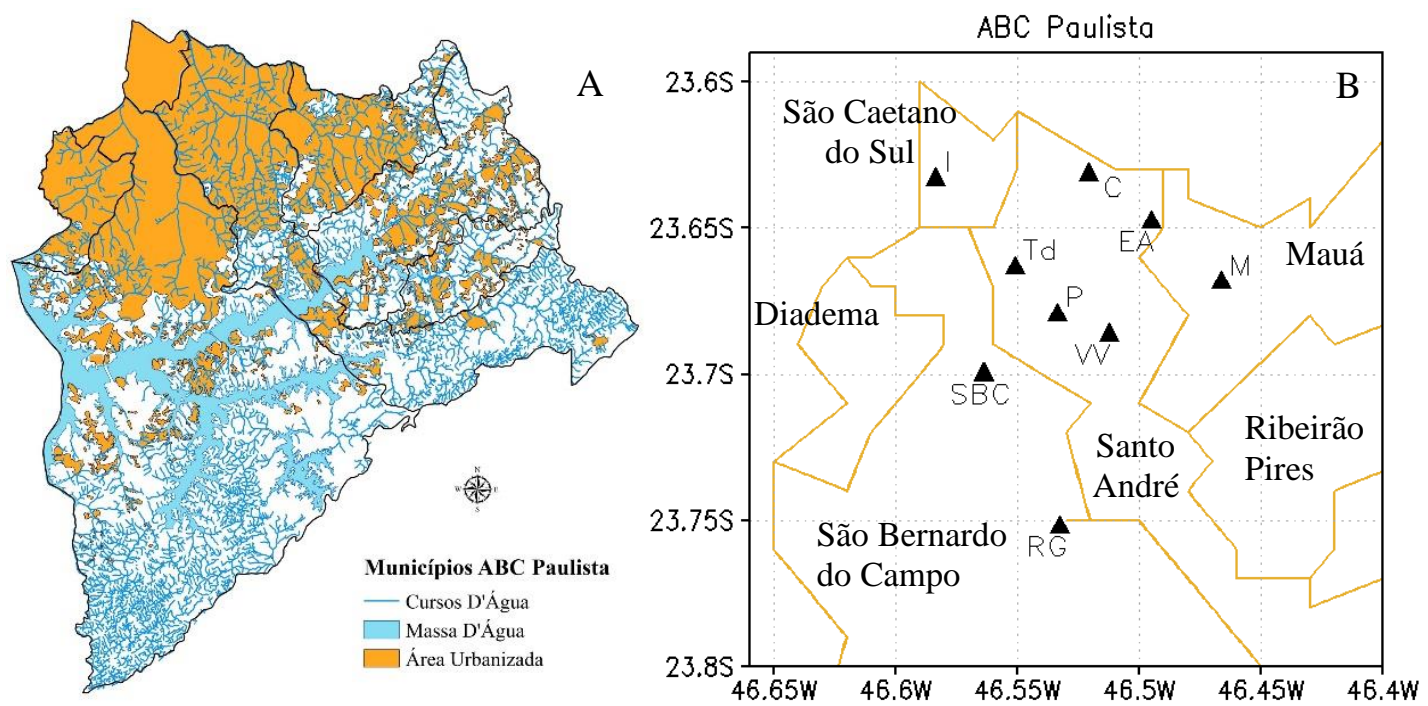

Figura 1. Mapa da região do ABC Paulista (A), e mapa com a localização dos pontos das estações meteorológicas (B): Santo André (Camilópolis - C, Erasmo Asunção - EA, Tanque de Detenção - Td, Paraiso - P e Vila Vitoria - VV), São Caetano do Sul (Ipiranga - I), São Bernardo do Campo (São Bernardo do Campo - SBC e Riacho Grande - RG) e Mauá ( Mauá - M).

O EMME realiza a alocação da matriz modelada de viagens da RMSP para o horário mais crítico do sistema que ocorre no período entre $7 \mathrm{~h}$ e $8 \mathrm{~h}$. Logo, os resultados que são apresentados nesse trabalho representam os trechos do sistema com a maior taxa de emissões no horário de pico da manhã.

A quantificação das emissões foi realizada apenas para destacar as vias de interesse para essa pesquisa e optou-se pelo método nacional de cálculo mais simples, mas sensível às condições de tráfego simuladas pelo modelo EMME. Logo, o método de modelagem de emissões de fontes móveis empregado é um método baseado em segmentos viários usando simulação determinística de tráfego para gerar os dados de fluxo e velocidade necessários para as equações de emissões propostas pelo IPEA/ANTP (1998).

Estimativas mais adequadas ao estado atual de desenvolvimento tecnológico de combustíveis e veículos bem como ao estado de conservação da frota podem ser obtidos usando o mesmo modelo de tráfego através do método MCT/MMA para veículos leves (Duarte e Paiva, 2013) e o método STAQ para veículos pesados (SISTRAN Engenharia, INECO e INECO do Brasil, 2014). Porém, esses métodos não são sensíveis às variações na velocidade do tráfego ou o ciclo de operação do motor.
Finalmente, através da comparação das informações dos mapas georeferenciados dos municípios do $\mathrm{ABC}$ com o mapa do modelo de simulação de transportes da CPTM (EMME), são observadas as possíveis correlações entre o sistema viário real e simulado e identificados os corredores de transportes e regiões com o maior potencial de emissões por fontes móveis. Porém, esse é um processo ainda em evolução, dado que os modelos de macro simulação da RMSP são mais acurados no município de São Paulo do que nas cidades periféricas.

Para a análise dos cenários futuros de temperatura na área urbana do ABC Paulista utilizaram-se três índices de temperatura derivados de dois modelos climáticos globais disponibilizados pelo Coupled Model Intercomparison Project Phase (CMIP5). O período da análise compreendeu o clima presente (1979-2005) e projeções futuras (2017-2040 20412070 e 2071-2100) para os cenários de emissões RCP2.6 e RCP8.5 (IPCC, 2013). No cenário mais otimista RCP2.6, projeta-se que o sistema terrestre armazenará $2,6 \mathrm{~W} / \mathrm{m}^{2}$ adicionais com uma concentração de $\mathrm{CO}_{2}$ em torno de 650 ppm, e para o cenário mais pessimista RCP8.5 o armazenamento adicional será de $8,5 \mathrm{~W} / \mathrm{m}^{2}$ e a concentração de $\mathrm{CO}_{2}$ equivalente a $1.370 \mathrm{ppm}$ (IPCC, 2013).

Os modelos climáticos selecionados foram os modelos japonês MRI-CGM3 (Meteorological Research Institute 
Coupled General Circulation Model - version 3) e francês CNRM-CM5 (Centre National de Recherches Météorologiques) disponibilizados pelo projeto Coupled Model Intercomparison Project (CMIP5) para o quinto relatório do IPCC, devido a possuir a melhor resolução espacial, em relação aos outros modelos climáticos de cenários futuros (IPCC, 2013).

Os índices de temperatura utilizados foram a maior temperatura mínima anual (TNx), a menor temperatura máxima anual (TXn) e a maior temperatura máxima anual (TXx).

Os modelos climáticos foram validados para o tempo presente (1979-2005) tendo como base a Reanálise - ERA Interim do European Centre for Medium-Range Weather Forecasts (ECMWF) (DEE et al., 2011), considerada neste estudo como a verdade terrestre, uma vez que na região de estudo não existe um histórico de dados de temperatura de no mínimo 30 anos. A métrica de validação foi o cálculo do erro sistemático

\section{Resultados e Discussão}

\section{Caraterísticas térmicas da região de estudo}

A Figura 2 mostra a espacialização da temperatura média do ar para os três meses do verão de 2014-2015 (dezembro 2014, janeiro 2015 e fevereiro 2015). Verifica-se que as áreas mais quentes se localizam preferencialmente sobre o norte do ABC Paulista, nos municípios de São Caetano do Sul (SCS), norte e noroeste da região urbana de Santo André (SA) e São Bernardo do Campo (SBC). Para o verão analisado, o mês de janeiro de 2015 foi o mês mais quente, seguido pelo mês de fevereiro 2015, tendo como áreas mais quentes o norte de SA e quase toda a área de SCS. Também, percebe-se nos três meses um núcleo mais frio sobre o sudeste de SBC, especificamente sobre o bairro rural Riacho Grande (Fig. 1B), mostrando uma diferença clara entre as temperaturas (aproximadamente $3^{\circ} \mathrm{C}$ ) sobre áreas densamente urbanas e bairros rurais.

Desta forma, pode-se inferir que as áreas mais quentes consideradas como ilhas de calor se encontram sobre os municípios com alta densidade demográfica, localizados ao norte do ABC Paulista, como é o caso de SCS com 91,30 hab./ha (IBGE, 2010).

O parâmetro mais importante que caracteriza a ilha de calor é sua intensidade ou magnitude, em um horário determinado, entre a temperatura de um ponto da cidade, densamente construído, e outro em seu entorno ou no ambiente rural (Garcia, 1996; Porangaba, 2017). Segundo Oke (1987), o mais recomendável é identificar o $\Delta \mathrm{T}$ no ciclo diurno da temperatura, a fim de identificar em qual período do dia é mais intenso.

A Tabela 1 mostra o $\Delta \mathrm{T}$ para o ciclo diurno médio de cada mês do verão analisado. Os valores de $\Delta \mathrm{T}$ entre os diferentes pontos das estações meteorológicas foram divididos por setores, e confirma-se que os setores norte e noroeste do ABC são os mais quentes, pois apresentam $\Delta \mathrm{T}$ positivos (Tabela 1). Quando são comparados os pontos localizados nas áreas urbanas mais densificadas, na tentativa de identificar ilhas de calor intraurbana, verifica-se que as intensidades do $\Delta \mathrm{T}$ são menores a $2{ }^{\circ} \mathrm{C}$ mostrando núcleos de intensidade fraca, porém, quando o cálculo do $\Delta \mathrm{T}$ é entre os pontos das áreas urbanas $\mathrm{e}$ as áreas mais afastadas, próximas a áreas de proteção ambiental (único ponto localizado no bairro rural de Riacho Grande de $\mathrm{SBC}$ ), o $\Delta \mathrm{T}$ é mais intenso caracterizando uma IC de intensidade média, como foi o caso do $\Delta \mathrm{T}$ entre o ponto da estação meteorológica de Camilopolis (C) e Riacho Grande (RG), que foi de $3,6{ }^{\circ} \mathrm{C}$ no mês de Janeiro, sendo que essa amplitude ocorre com maior frequência no horário vespertino. Por outro lado, o setor nordeste (município de Mauá), se apresentou menos quente, sobretudo no mês de fevereiro com $\Delta$ Ts negativos. Já, no setor sul (Riacho Grande) os $\Delta$ Ts foram negativos nos três meses o que identifica ilhas de frescor.
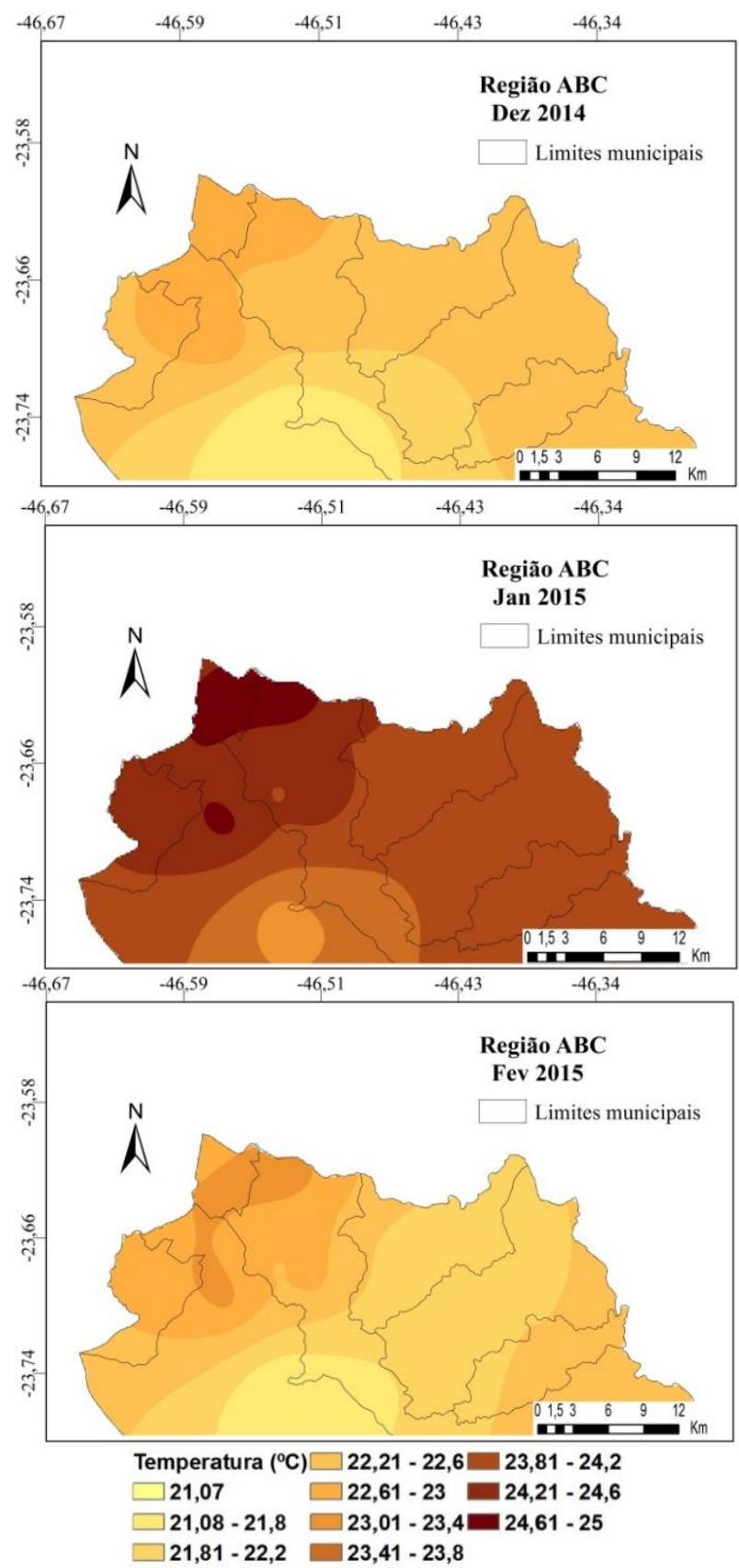

Figura 2. Espacialização da temperatura média mensal na região do $\mathrm{ABC}$ Paulista (São Paulo, Brasil) para os meses de dezembro de 2014, janeiro e fevereiro de 2015. 
Tabela 1. Magnitudes das intensidades das ilhas de calor urbanas atmosféricas obtidas através do $\Delta \mathrm{T}\left({ }^{\circ} \mathrm{C}\right)$ do ciclo diurno para os meses do verão 2014-2015, na área urbana do ABC Paulista.

\begin{tabular}{ccccc}
\hline Setor & Estações & Dez/2014 & Jan $/ 2015$ & Fev/2015 \\
\hline \multirow{5}{*}{ Norte } & C-VV & $1,2^{\text {icf }}$ & $1,3^{\text {icf }}$ & $1,1^{\text {icf }}$ \\
& C-P & $1,4^{\text {icf }}$ & $1,3^{\text {icf }}$ & $1,6^{\text {icf }}$ \\
& C-Td & $1,5^{\text {icf }}$ & $1,2^{\text {icf }}$ & $1,4^{\text {icf }}$ \\
& C-RG & $3,3^{\text {icm }}$ & $3,6^{\text {icm }}$ & $3,4^{\text {icm }}$ \\
& I-Td & $2,2^{\text {icm }}$ & $2,4^{\text {icm }}$ & $1,8^{\text {icm }}$ \\
\multirow{4}{*}{ Noroeste } & I-RG & $3,1^{\text {icm }}$ & $2,3^{\text {icm }}$ & $2,5^{\text {icm }}$ \\
\hline \multirow{4}{*}{ Nordeste } & SBC-C & $0,5^{\text {icf }}$ & $0.5^{\text {icf }}$ & $0,5^{\text {icf }}$ \\
& SBC-RG & $2,7^{\text {icm }}$ & $2,7^{\text {icm }}$ & $2,9^{\text {icm }}$ \\
& SBC-M & $1,3^{\text {icf }}$ & $1,7^{\text {icf }}$ & $2,0^{\text {icf }}$ \\
\hline \multirow{2}{*}{ Sul } & M-P & $1,9^{\text {icf }}$ & $2,0^{\text {icf }}$ & $-1,9^{\text {if }}$ \\
& M-C & $1,6^{\text {icf }}$ & $1,9^{\text {icf }}$ & $-2,4^{\text {if }}$ \\
& RG-VV & $-1,9^{\text {if }}$ & $0,7^{\text {icf }}$ & $-2,7^{\text {if }}$ \\
\hline & RG-M & $-3,4^{\text {if }}$ & $-3,3^{\text {if }}$ & $-3,3^{\text {if }}$ \\
\hline
\end{tabular}

Lgenda: if: Ilha de frescor $\left(\Delta \mathrm{T}<0{ }^{\circ} \mathrm{C}\right)$; icf: Ilha de calor de fraca magnitude $\left(0,1^{\circ} \mathrm{C}\right.$ a $\left.2,0^{\circ} \mathrm{C}\right)$; icm: Ilha de calor de média magnitude $\left(2,1{ }^{\circ} \mathrm{C} \mathrm{a} 4,0{ }^{\circ} \mathrm{C}\right)$.

Legenda: C-VV diferença de temperatura (DT) entre Camilópolis e Vila Vitória (SA); C-P DT entre Camilópolis e Paraíso (SA); C-Td DT entre Camilópolis e Tanque de Detenção (SA); C-RG DT entre Camilópolis (SA) e Riacho Grande (SBC); I-Td DT entre Ipiranga (SCS) e Tanque de Detenção (SA); I-RG DT entre Ipiranga (SCS) e Riacho Grande (SBC); SBC- C DT entre São Bernardo do Campo e Camilópolis (SA); SBC-RG DT entre São Bernardo do Campo e Riacho Grande; SBC-M DT entre São Bernardo do Campo e Mauá; M-C DT entre Mauá e Camilópolis (SA); M-P DT entre Mauá e Paraíso (SA); M-VV DT entre Mauá e Vila Vitória (SA); RG-VV DT entre Riacho Grande (SBC) e Vila Vitória (SA); RG-M DT entre Riacho Grande (SBC) e Mauá.

Valores de $\Delta \mathrm{T}$ que identificam intensidades de ilhas de calor variam de acordo com o tipo e uso de ocupação do solo, em cidades de São Paulo para dias específicos os valores chegam até $4,5^{\circ} \mathrm{C}$, como é o caso da cidade de Rancharia (Porangaba et al., 2017), e até $2,5{ }^{\circ} \mathrm{C}$ na região central da cidade de São Carlos (Vasconcelos et al., 2013). No entanto, é importante ressaltar que o valor de $\Delta \mathrm{T}$ obtido na presente pesquisa constitui um valor médio de um mês específico, e por ser um valor médio pode apresentar valores baixos, dependendo do mês e setor analisado, ou valores altos como foi o caso do mês de janeiro de 2015.

\section{Análise da simulação de tráfego e das emissões no $A B C$ Paulista}

Os resultados desta seção são baseados no modelo de simulação de tráfego EMME/CPTM para o cenário 2014. Nas figuras 3 e 4 são identificados os trechos do sistema viário principal do $\mathrm{ABC}$ paulista com as maiores taxas de emissões por quilômetro de via. Na Figura $3 \mathrm{~A}$ as espessuras das barras vermelhas indicam as intensidades de emissões de $\mathrm{CO}_{2} / \mathrm{km}$ nos trechos do modelo. Na Figura 3B pode ser observado com mais detalhe as grandes avenidas (Dos Estados, Industrial, Dom Pedro II, etc) da área urbana de Santo André que apresentam as maiores taxas de emissões.

Cabe destacar que sem um modelo de emissões que considere ao menos a velocidade de tráfego em sua estrutura de cálculo, a construção desse mapa não seria possível. Os picos de emissões no horário das $7 \mathrm{~h}$ às $8 \mathrm{~h}$ correspondem aos trechos do sistema viário de maior lentidão devido aos congestionamentos das vias cuja capacidade foi atingida ou superada pelo fluxo de veículos, simulado pelo modelo de alocação de tráfego da RMSP no cenário de 2014.

O modelo de redes exemplificado na Figura 3A foi testado com três métodos de cálculo de emissões: IPEA/ANTP (sensíveis ao fluxo e velocidade dos veículos), MCT/MMA (sensível ao fluxo, idade e tecnologia dos veículos) e o método International Vehicle Emission (sensível ao fluxo, velocidade dos veículos, tecnologia e condições climáticas) e comparado ao estudo desenvolvido pelo WRI/ICLEI empregando o GHG Protocol (sensível à idade e tecnologia dos veículos) (CIGABC, 2016).

A Tabela 2 mostra o resultado das simulações para cada município do ABC Paulista, de quatro modelos aplicados no que se refere às estimativas de emissões de $\mathrm{CO}_{2}$. Como pode ser observado nessa tabela, todos os métodos divergem na quantificação da massa de $\mathrm{CO}_{2}$, tornando muito incerta as estimativas, mas todos concordam quanto à identificação dos municípios de maior emissão.

Apesar dos resultados da Tabela 2 (Paiva, 2017), agregarem as emissões de municípios inteiros, salvo o WRI/ICLEI (CIGABC, 2016), todos os demais foram aplicados através de um modelo de redes de transportes e podem produzir resultados para pequenas áreas de cada município, bem como para as vias e corredores de transportes e representar os fluxos de veículos de passagem. A única ressalva à utilização desses modelos é a atualização de seus coeficientes de emissões. O IPEA/ANTP emprega fatores de emissão do final dos anos 90 e o IVE usa fatores que deixaram de ser atualizados em 2010. Por essa razão esses dois últimos métodos tendem a superestimar as emissões.

Dentre os métodos apresentados, o MCT/MMA apesar de não ser sensível às variações de velocidade do tráfego, como ocorre com o IPEA/ANTP e IVE, apresenta estimativas com uma grandeza provavelmente mais próxima à realidade, porque possibilita a aplicação de fatores de emissões atualizados para o contexto nacional (Duarte e Paiva, 2013) em modelos de redes de transportes convertendo os fluxos simulados em emissões por segmento viário da rede do município, construindo dessa forma mapas de emissões com uma grandeza adequada para análises desagregadas.

Logo, enquanto não for disponibilizado um modelo de emissões nacional preparado para trabalhar com modelos de simulação de tráfego, a abordagem sugerida é o uso combinado de modelos, um para localizar áreas de maior potencial de emissões e outro para quantificá-las de forma mais realista.

Cabe destacar aqui que os resultados obtidos por esses métodos não permitem de forma trivial uma associação direta das emissões estimadas com a qualidade do ar ou possíveis alterações climáticas observadas na região de estudo, devido à complexidade de interações envolvidas em cada um desses fenômenos.

Note que na Tabela 2 as emissões municipais são calculadas considerando a extensão viária de cada município a fim de identificar as malhas de maior potencial de emissões. 


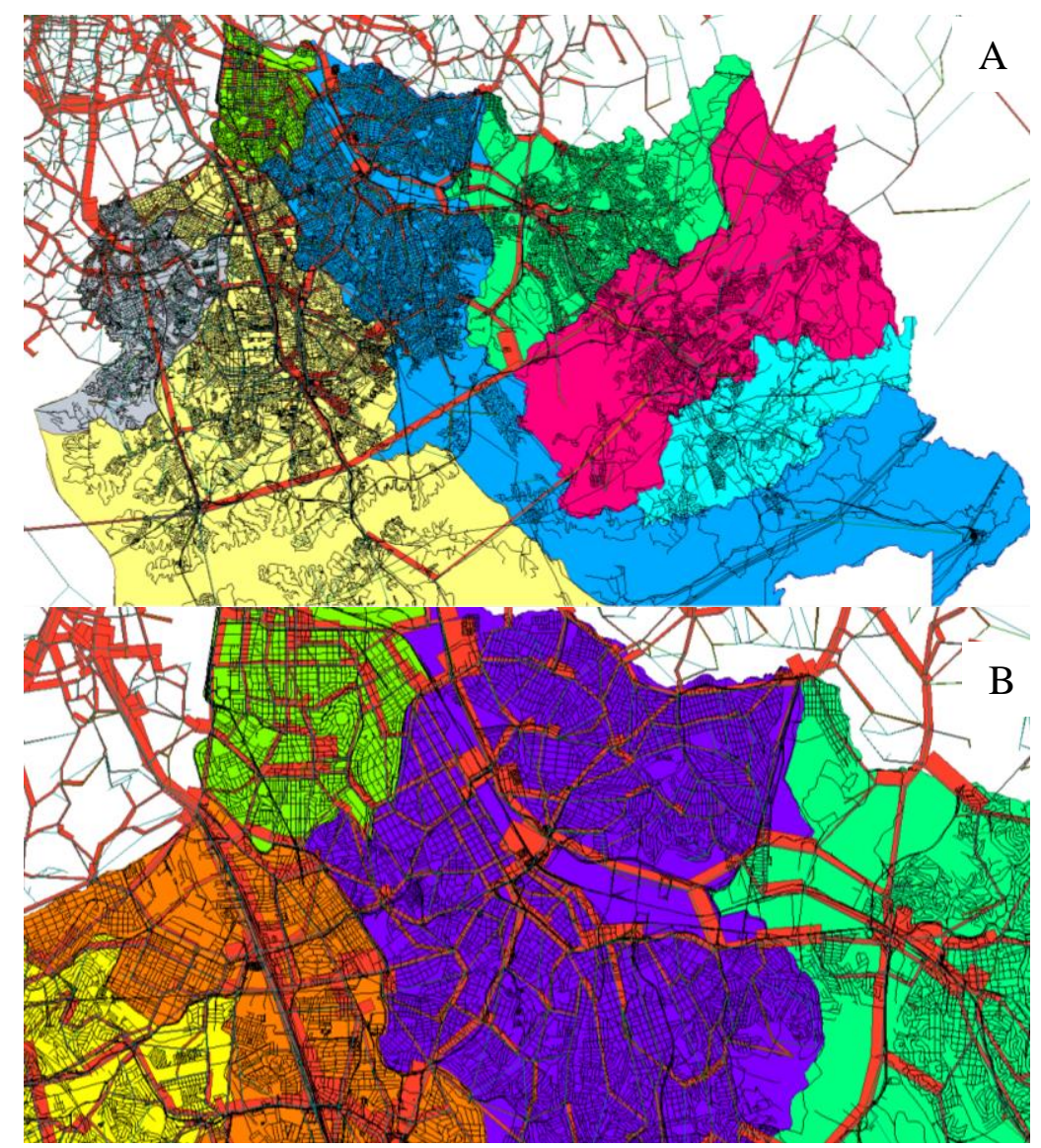

Figura 3. Trechos do sistema viário com maiores taxas de emissões de $\mathrm{CO}_{2} / \mathrm{km}$ na região urbanizada dos municípios do $\mathrm{ABC}$ paulista. A: Da esquerda para direita são representados os municípios de Diadema, São Bernardo do Campo, São Caetano do Sul, Santo André, Mauá, Ribeirão Pires e Rio Grande da Serra). B: Detalhe da área central do município de Santo André (azul).

Tabela 2. Comparação de métodos de estimativa de emissões por km viário.

\begin{tabular}{lcccccccc}
\hline & \multirow{2}{*}{$\begin{array}{c}\text { Município } \\
\text { Viária }(\mathrm{km})\end{array}$} & Frota (autos) & \multirow{2}{*}{$\begin{array}{c}\text { VM } \\
(\mathrm{km} / \mathrm{h})\end{array}$} & Veiculo*(km) & & \multicolumn{4}{c}{$\mathrm{CO}_{2}$ (ton/km viário) } \\
\hline S. Bernardo do Campo & & ANTP & IVE & WRI/ & MCT/ \\
Santo André & 1179 & 380.166 & 33,6 & 908.187 & 0,279 & 0,177 & 0,109 & 0,131 \\
Mauá & 1253 & 352.905 & 32,2 & 385.296 & 0,169 & 0,108 & 0,095 & 0,052 \\
Diadema & 533 & 135.556 & 31,6 & 183.555 & 0,205 & 0,131 & 0,086 & 0,058 \\
S. Caetano do Sul & 372 & 119.182 & 33,4 & 192.675 & 0,219 & 0,139 & 0,112 & 0,088 \\
Ribeirão Pires & 243 & 99.921 & 25,6 & 116.970 & 0,329 & 0,203 & 0,138 & 0,082 \\
Rio Grande da Serra & 525 & 43.228 & 38,4 & 208.298 & 0,092 & 0,058 & 0,027 & 0,067 \\
ABC Paulista & 133 & 11.169 & 49,7 & 8.449 & 0,014 & 0,009 & 0,028 & 0,011 \\
\hline
\end{tabular}

Legenda: VM - velocidade média; IPEA - Instituto de pesquisa econômicaaplicada; ANTP - Associação nacional de transporte público; IVE - International Vehicle Emission; WRI - World Resources Institute; ICLEI - International Council for Local Environmental Initiatives; MCT - Ministério da Ciência e Tecnologia; MMA - Ministério do Meio Ambiente.

Com esse indicador de desempenho as malhas mais densas se destacam quanto às emissões específicas facilitando a comparação com as ilhas de calor da região.

Discussão do campo térmico no ABC Paulista e da simulação de tráfego e das emissões de $\mathrm{CO}_{2}$

As pesquisas divulgadas pelos relatórios do IPCC demonstram uma relação direta entre $\mathrm{o}$ aumento da temperatura do ar e o aumento nas concentrações de $\mathrm{CO}_{2}$ (IPCC, 2007 e 2013). No caso específico desse trabalho, as emissões devido ao sistema viário do $\mathrm{ABC}$ paulista foram simuladas como explicado na seção anterior, e é possível observar relações importantes entre áreas mais quentes do ABC Paulista e as de maior concentração de $\mathrm{CO}_{2}$ devido ao tráfego.

Observou-se que o município de São Caetano do Sul e os setores norte de Santo André e de São Bernardo do Campo são 
os que apresentam as mais altas temperaturas no verão analisado. Dentre esses municípios, São Bernardo do Campo e Santo André se destacam como os de maior emissão de $\mathrm{CO}_{2}$ (Tabela 2). Estes municípios apresentam a maior área urbana do ABC Paulista e possuem extensas e movimentadas rodovias, como pode ser observado na Figura 3A. A Avenida dos Estados que cruza Santo André (Figura 3B), a Avenida Anchieta que cruza São Bernardo do Campo, e o Rodoanel Mário Covas que atravessa os dois municípios, estão entre as principais vias de tráfego do ABC Paulista.

Por outro lado, o resultado mais detalhado de identificação das malhas de maior potencial de emissões (Tabela 2) conseguiu capturar informações mais específicas, sendo que na simulação dos modelos IPEA/ANTP, IVE e WRI/ICLEI, os municípios de São Caetano de Sul e Diadema tiveram destaque, sendo São Caetano do Sul o que apresentou uma maior quantidade de emissões por ton $/ \mathrm{km}$ viário. Estes municípios se destacaram também como as áreas mais quentes, principalmente São Caetano do Sul, embora tenha a menor extensão de todos os municípios do ABC Paulista, porém como mostrado na Figura $3 \mathrm{~B}$ a maioria dos trechos do sistema viário apresentam espessuras consideráveis ao longo de toda a sua área.

Vale ressaltar que as emissões de GEE também provem de outras fontes além do tráfego no ABC Paulista. Há a densificação das áreas centrais com a presença de indústrias em torno dos principais eixos de transporte, em especial, a Rhodia, o Polo Petroquímico de Capuava e a Pirelli.

Nessa primeira análise foi possível encontrar associações diretas entre o campo térmico e as emissões de $\mathrm{CO}_{2}$ obtidas das simulações dos modelos de tráfego no ABC Paulista.

\section{Cenários climáticos futuros de temperatura para a região urbana do ABC Paulista}

No contexto de já existirem cenários da distribuição do veículo*km anual nacional por tipo de frota de automóveis em diferentes horizontes com tendência de aumento para a Frota Flex Etanol e Frota Flex Gasolina para o cenário 2020 (Duarte e Paiva, 2013), espera-se que esse cenário influencie no aumento da temperatura.

Com o intuito de quantificar esse aumento de temperatura, nessa seção se analisam índices de temperatura para dois modelos climáticos, já detalhados na metodologia, e para dois cenários de emissões futuros: RCP2.6 e RCP8.5, na região urbana do ABC Paulista.

Os índices selecionados para a análise mostram o comportamento da temperatura máxima e mínima na escala anual para períodos de 23 e 30 anos. Os resultados são mostrados em anomalias (Tabela 3), que representam as diferenças entre o clima atual (1961-1990) e os cenários futuros, quando positivo o valor significa que existirá um aumento, e quando negativo que existirá uma diminuição segundo a simulação do modelo.

A Tabela 3 mostra o resultado das anomalias, sendo positivas para todos os cenários e modelos analisados (MRICGM3 e CNRM-CM5) indicando aumentos em relação ao padrão médio climatológico (1961-1990).
Tabela 3. Anomalias $\left({ }^{\circ} \mathrm{C}\right)$ dos índices de maior temperatura máxima anual (TXx), menor temperatura máxima anual (TXn) e maior temperatura mínima anual (TNx) simuladas pelos modelos MRI-CGM3 e CNRM-CM5 para os cenários de emissões RCP2.6 e RCP8.5.

\begin{tabular}{ccccccc}
\hline \multirow{2}{*}{$\begin{array}{c}\text { Período de } \\
\text { análise }\end{array}$} & \multicolumn{2}{c}{ TXx } & \multicolumn{2}{c}{ TXn } & \multicolumn{2}{c}{ TNx } \\
\cline { 2 - 8 } & MRI & CNRM & MRI & CNRM & MRI & CNRM \\
& CGM3 & CM5 & CGM3 & CM5 & CGM3 & CM5 \\
\hline $2018-2040$ & 0,77 & 1,87 & 0,94 & 0,29 & 0,80 & 1,01 \\
$2011-2040$ & 0,66 & 2.04 & 0,78 & 0,21 & 0,70 & 0,9 \\
$2041-2070$ & 0,88 & 2,22 & 0,83 & 0,57 & 0,67 & 1,30 \\
2071-2100 & 1,17 & 2,22 & 0,61 & 0,96 & 1,05 & 1,24 \\
\hline \multirow{2}{*yyyyyyy}{ Período de } & \multicolumn{2}{c}{ TXx } & \multicolumn{2}{c}{ TCP8,5 } & & \\
\cline { 2 - 8 } análise & MRI & CNRM & MRI & CNRM & MRI & CNRM \\
& CGM3 & CM5 & CGM3 & CM5 & CGM3 & CM5 \\
\hline 2018-2040 & 0,56 & 1,86 & 0,49 & 1,11 & 0,61 & 0,88 \\
$2011-2040$ & 0,59 & 1,29 & 0,42 & 0,79 & 0,58 & 0,86 \\
$2041-2070$ & 2,42 & 3,49 & 1,63 & 1,81 & 2,10 & 2,04 \\
$2071-2100$ & 2,85 & 6,34 & 2,23 & 2,95 & 2,82 & 3,27 \\
\hline
\end{tabular}

Para o cenário mais otimista (RCP2.6) as anomalias positivas chegam até $2,2{ }^{\circ} \mathrm{C}$, e crescem gradualmente desde o período 2018-2040 até 2071-2100. A maior temperatura máxima anual (TXx) é a que sofrerá o maior incremento (> 2 ${ }^{\circ} \mathrm{C}$ ), sendo o modelo CNRM-CM5 o que simula os mais altos valores.

Já para o cenário pessimista RCP8.5 as anomalias positivas se incrementam sobretudo para os períodos 2041-2070 e 20712100, sendo novamente o modelo CNRM-CM5 que simula as maiores anomalias, um aumento na temperatura máxima anual de $3,49{ }^{\circ} \mathrm{C}$ para o período $2041-2070$ e de $6,34{ }^{\circ} \mathrm{C}$ para o período de 2071-2100. O índice que descreve a maior temperatura mínima anual (TNx) também sofrerá um aumento não tão intenso como o TXx, mas chega a ser de $3,27{ }^{\circ} \mathrm{C}$ no período de 2071-2100.

A Figura 4 mostra a variabilidade temporal anual do índice da maior temperatura máxima anual (TXx) para o clima presente (1961-1990) e para os períodos de 2011 até 2100 simulados pelos modelos MRI-CGM3 (Fig. 5a) e CNRMCM5(Fig. 5b).

Observa-se uma alta variabilidade da temperatura do ar, tanto no período histórico como nos cenários RCP2.6 e RCP8.5, sendo mais intensa no modelo CNRM-CM5, como já retratado nas anomalias. Também é clara a tendência de aumento ao longo dos anos, sendo mais intensa para o cenário pessimista RCP8.5 e para o período de 2071-2100.

O erro sistemático foi menor para o modelo CNRM-CM5 tendo como referência os dados da Reanálise - ERA Interim.

\section{Conclusões}

A pesquisa mostrou claras associações entre as áreas mais quentes, as mais densamente urbanizadas, e as vias com a maior quantidade de emissões de $\mathrm{CO}_{2}$ por ton $/ \mathrm{km}$, sendo os municípios de São Caetano do Sul, norte de Santo André e norte de São Bernardo do Campo, que se apresentaram como os mais quentes, indicando uma ilha de calor de média intensidade $\left(3,6^{\circ} \mathrm{C}\right)$. 

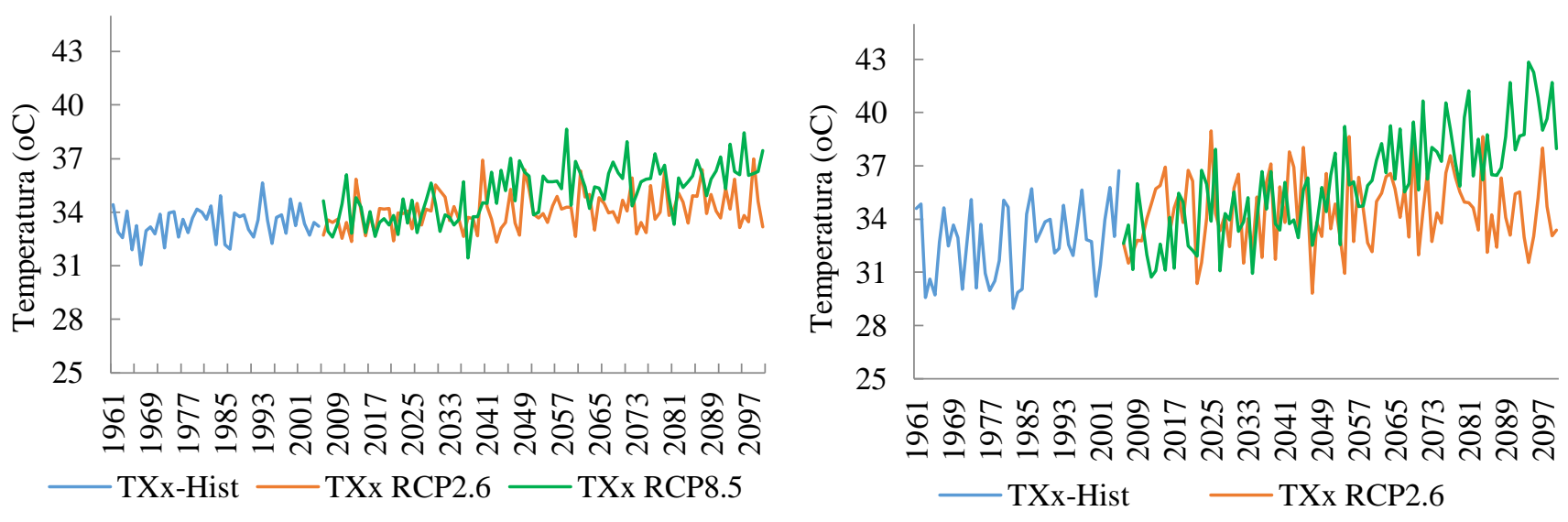

Figura 4. Gráficos da variabilidade anual da temperatura máxima anual para os modelos MRI-CGM3 (A) e para o modelo CNRM-CM5 (B) para o periodo 1961-2100 para os cenários RCP2.6 e RCP8.5

Por outro lado, os cenários dos modelos climáticos, apesar das incertezas, mostraram aumento na temperatura máxima e mínima anual, trazendo um cenário de dias e noites mais quente, com um aumento de $6,34{ }^{\circ} \mathrm{C}$ para o cenário RCP8,5 no período de 2071-2100. Diante do encontrado na presente pesquisa, as medidas de mitigação no que se refere ao controle de emissões de $\mathrm{CO}_{2}$ pelo setor transporte no $\mathrm{ABC}$ Paulista, devem incluir políticas públicas do transporte motorizado em conjunto com restrições de uso e ocupação do solo urbano, bem como a adoção de novas tecnologias veiculares e sistemas de mobilidade, pois os impactos, no aumento da temperatura, e diante de um cenário de mudança climática tendem a se incrementar colocando em risco a qualidade de vida e saúde pública, bem como e o meio ambiente.

\section{Agradecimentos}

Os Autores gostariam de agradecer a Fundação de Amparo à Pesquisa do Estado de São Paulo - FAPESP (PROCESSO 2016/14563-5) pelo apoio recebido.

\section{Referências}

Boian, C.; Andrade, M.F.; 2012. Characterization of ozone transport among metropolitan regions. Revista Brasileira de Meteorologia, 27: 229-242.

CETESB; 2016. Qualidade do ar no estado de São Paulo 2015. São Paulo, CETESB, 165 p.: il. color.

Ccoyllo, O.R.S.; Andrade, M.F. 2002. The influence of meteorological conditions on the behavior of pollution concentration in São Paulo, Brazil. Environmental Pollution, 116(2) 257-263.

Dee, D.P. et al., 2011. The ERA-Interim reanalysis: configuration and performance of the data assimilation system. Quarterly Journal of the Royal Meteorological Society, 137(656) 553-597.

CIGABC; $20161^{\circ}$ Inventário Regional. Emissões de Gases de Efeito Estufa do Grande ABC. Consórcio Intermunicipal Grande ABC, ICLEI. Santo André.

Duarte, L.H.K. Paiva, H. 2013. Inventário de emissões por fontes móveis em cidades de pequeno e médio porte. In: Tobias, M.S.G. e Lima, A.C.M. Urbanização \& Meio Ambiente. Belém. Unama - Universidade da Amazônia. 451-436p.

Freitas, E.D.; Silva Dias, P.L; Rozoff, C.M; Cotton, W.R. 2007. Interactions of an urban heat island and sea-breeze circulations during winter over the metropolitan area of Sao Paulo, Brazil. Boundary-Layer Meteorology, 122(1) 43-65.
IPEA e ANTP. 1998. Redução das deseconomias urbanas com a melhoria do transporte público. Relatório síntese. Instituto de pesquisa econômica aplicada e associação nacional de transportes públicos. Brasília, Brasil.

Intergovernmental Panel on Climate Change - IPCC. 2013: Climate Change 2013: The Physical Science Basis. Contribution of Working Group I to the Fifth Assessment Report of the Intergovernmental Panel on Climate Change [Stocker, T.F., D. Qin, G.K. Plattner, M. Tignor, S.K. Allen, J. Boschung, A. Nauels, Y. Xia, V. Bex and P.M. Midgley (eds.)]. Cambridge University Press, Cambridge, United Kingdom and New York, NY, USA, 1535 pp.

Lombardo, M.A. 1985. Ilhas de calor nas metrópoles: o exemplo de São Paulo. Hucitec, São Paulo, 244p.

Martins, L.D.; Andrade, M.F. 2008. Ozone Formation Potentials of Volatile Organic Compounds and Ozone Sensitivity to Their Emission in the Megacity of Sao Paulo, Brazil. Water, Air and Soil Pollution, 195(1-4) 201-213.

Oke, T. R. 1987. Street design and urban canopy layer climate. Energy and Buildings, 11(1-3) 103-113.

Paiva. H.; 2017. Mudanças Climáticas e Inventários Regionais de Emissões. $21^{\circ}$ Congresso Brasileiro de Transporte e Trânsito. Associação Nacional de Transportes Públicos. São Paulo.

Porangaba, G.F.O; Teixeira, D.C.F; Amorim, M.C. 2017. Procedimentos Metodológicos para análise das ilhas de calor em cidades de pequeno e médio porte. Revista Brasileira de Climatologia, 21(13) 225-247.

Randall, D.A. et al. 2007. In: Climate Change 2007: The Physical Science Basis. Contribution of Working Group I to the Fourth Assessment Report of the Intergovernmental Panel on Climate Change [Solomon, S., D. Qin, M. Manning, Z. Chen, M. Marquis, K.B. Averyt, M.Tignor and H.L. Miller (eds.)]. Cambridge University Press, Cambridge, United Kingdom and New York, NY, USA.

SISTRAN Engenharia INECO e INECO do Brasil; 2014. Estudo Comparativo de Tecnologias Veiculares de Tração Aplicáveis a Ônibus Urbanos. Série Cadernos Técnicos. Volume 15. São Paulo. ANTP, 88p.

Stull, R.B. 1988. An Introduction to Boundary Layer Meteorology. Ed. Kluwer Academic Publishers, Dordrecht, Boston and London, 666p.

Vasconcellos, J.S.; Souza, L.C.L.; Teixeira, B.A. 2013. Comportamento térmico em uma fração urbana no município de São Carlos. In: IV Congresso Brasileiro de Gestão Ambiental, Salvador, Bahia, Brasil. 